\title{
Measuring voluntary activation of the Quadriceps femoris during isokinetic concentric contractions
}

\author{
Chiara Malloggi $^{\mathrm{a}}$, Luigi Catino ${ }^{\mathrm{b}}$, Viviana Rota ${ }^{\mathrm{a}}$, Stefano Scarano ${ }^{\mathrm{a}}$ and Luigi Tesio ${ }^{\mathrm{a}, \mathrm{b}}$ \\ ${ }^{a}$ Department of Neurorehabilitation Sciences, Istituto Auxologico Italiano, IRCCS, Ospedale San Luca, Milan, \\ Italy \\ ${ }^{\mathrm{b}}$ Department of Biomedical Sciences for Health, Università degli Studi di Milano, Milan, Italy
}

Received 20 December 2018

Accepted 28 February 2019

\begin{abstract}
.
BACKGROUND: It is known that Voluntary Activation (VA) of muscles may be lower during isokinetic concentric (CON) contractions than during isometric (ISOM) contractions, and that it may be further decreased in various motor impairments.

OBJECTIVE: The aim of this study was to validate the Interpolated Twitch Technique (ITT) for quantifying VA of the Quadriceps femoris during CON contractions (knee extension at 60 and $120^{\circ} / \mathrm{s}$, CON60 and CON120, respectively).

METHODS: Pairs of electrical stimuli were delivered to the Quadriceps femoris of twenty-two healthy subjects at $50^{\circ}$ of knee flexion. Participants were instructed to exert five different levels of effort in decreasing order between $100 \%$ and $20 \%$ maximal voluntary efforts in ISOM and CON contractions.

RESULTS: Through a linear regression model, a significant relationship between measures of VA and moment was observed for all the three contraction conditions: slopes (95\% confidence intervals) $=1.04(0.98-1.11), 0.96(0.89-1.02)$ and $0.84(0.78-0.91)$; intercepts $=-5.22(-8.61--1.83), 4.16(0.71-7.62)$ and $14.58(10.76-18.39)$, for ISOM, CON60 and CON120 contractions, respectively. This supported the validity of the method.

CONCLUSIONS: It is concluded that ITT can be a valid method for measuring VA during CON contractions, potentially useful
\end{abstract} both in sports and rehabilitation studies.

Keywords: Twitch interpolation technique, voluntary activation, knee extension, isokinetic concentric contraction, quadriceps femoris

\section{Introduction}

Voluntary activation (VA) can be defined as the amount of recruitment of muscles during a voluntary contraction effort. The number of spinal motoneurones recruited and their firing frequency increase with increasing VA [1]. The assessment of VA may be rele-

\footnotetext{
*Corresponding author: Luigi Tesio, Department of Neurorehabilitation Sciences, Istituto Auxologico Italiano, IRCCS, Ospedale San Luca, via Mercalli 32, Milan 20122, Italy. Tel.: +39 258218 151; E-mail: luigi.tesio@unimi.it,1.tesio@auxologico.it.
}

vant in patients affected by orthopedic and/or neurological disorders, as long as it may disentangle central from peripheral causes of weakness. VA failure has been reported to be caused by lesions of the central nervous system or inhibitory stimuli arising from peripheral damaged tissues ("arthrogenous muscle weakness") [2,3]; it may also represent an unconscious form of prevention of postural imbalance [4].

The "interpolated twitch technique" (ITT) is the most widely used method to quantify the extent of VA [5]. It consists in stimulating a representative (i.e., greater than 20\%) sample of the muscle belly through 
an electric shock both during a voluntary contraction and at rest. The shock is painless, although it may cause some discomfort, associated with the unexpected strong muscle contraction. If the shock does not generate an extra force during contraction, all muscle fibers belonging to the sample reached by the electric shock can be claimed to be recruited at their tetanic frequency. Otherwise, following the stimulus a twitch can be observed [6-8] revealing that the voluntary command was only partially effective in recruiting the available pool of spinal motoneurones.

Previous studies focused on the study of VA in various upper [5,9] and lower [7,10] limb muscles. Regarding the Quadriceps femoris, isometric (ISOM) contractions were mostly investigated. Contrary to common "isotonic" contractions, where load is constant but joint rotation speed changes, ISOM contractions can be supposed to be quite infrequent in daily life. Also, VA and joint angular velocity are linked by complex relationships. The discharge rate of spindle endings, facilitating motoneurones' recruitment, decreases during shortening contractions, compared to ISOM contractions. Spindle-based facilitation should be lower, the higher the shortening speed, whereas the opposite should be true for force-sensitive joint receptors. The overall balance seems that of a lower motoneuron excitation during the end part of knee extension contractions $[11,12]$. As a further complication, speeddependence of moment arises from the individual stiffness of the series elastic components. At a given joint angle, the overall length reached by the muscle-tendon complex relies more on shortening of the elastic components, compared to muscle fibers, the higher the shortening speed [13]. Slack of the elastic element (following reduced mobility or aging itself, and little predictable) may involve greater fiber shortening, thus adding to the loss of spindle-mediated facilitation at higher rotation speed. A decreased VA may thus ensue due to both mechanical and neural causes (e.g. central nervous lesions or pain-dependent inhibition $[2,14]$ ).

Imposing stable and predetermined joint angular velocity still is the ideal method to study empirically the relationship between force and VA during muscle shortening. Isokinetic machines provide a good solution, in that they impose a fixed rotation speed to the subject's joint, whichever the moment applied [15]. This notwithstanding, investigations on the relationship between VA and joint rotation speed during isokinetic concentric (CON) contractions are very sparse and provide conflicting results. Some authors have concluded that maximal $\mathrm{CON}$ voluntary activation did not differ significantly from the maximal ISOM level either at slow $(20 \%$ s) or at faster angular velocities $\left(150\right.$ and $\left.300^{\circ} / \mathrm{s}\right)[9,16]$. Other found that the mean activation levels during maximal eccentric and maximal CON contractions were significantly lower with respect to maximal ISOM contractions [17].

To the best of the Author's knowledge, the relationship between twitch amplitude and VA, expected to be linear, has been widely explored during ISOM contractions [18-20], but only at slow angular velocities $(20 \%$ s) during CON contractions [17].

The aim of the present study was to validate the ITT on the Quadriceps femoris during CON contractions using pairs ("doublets") of electrical stimuli at intermediate angular velocities $\left(60^{\circ} / \mathrm{s}\right.$ and $120^{\circ} / \mathrm{s}$, CON60 and CON120, respectively). As an index of validity of the method, the linearity of the relationship between measures of VA and measures of moments was tested at various levels of voluntary effort in a sample of healthy adults.

\section{Method}

\subsection{Participants}

The goal of the study was to test the validity of the method, rather than providing normative values. Therefore, a sequential design was chosen [21,22]. The objective was to find a satisfactory linear regression model linking VA to voluntary moment, for each angular velocity. A Pearson correlation $r>0.9$ (variance explanation $R^{2}>0.81$ ), with both slope and intercept significant at $p<0.05$, was the predetermined aim with a sample size of at least 10 subjects per contraction condition (ISOM, CON60, and CON120). This implied that more than 10 subjects had to be enrolled, because not all subjects could provide valid data for all levels of effort and contraction conditions (see below, Data processing and analysis).

The inclusion criteria were: i) age between 18 and 45 years; ii) ability to wittingly sign the informed consent form; iii) ability to understand the instructions and to complete the motor task. All subjects had to be recreationally active. Subjects were excluded if they had a history of epilepsy (to avoid the risk for seizures triggered by the stimuli), implanted electrosensitive devices, or had any neurologic condition or orthopedic condition limiting the articular mobility or muscular strength of the lower limbs. No subject under treatment with oral anticoagulant or antiplatelet 


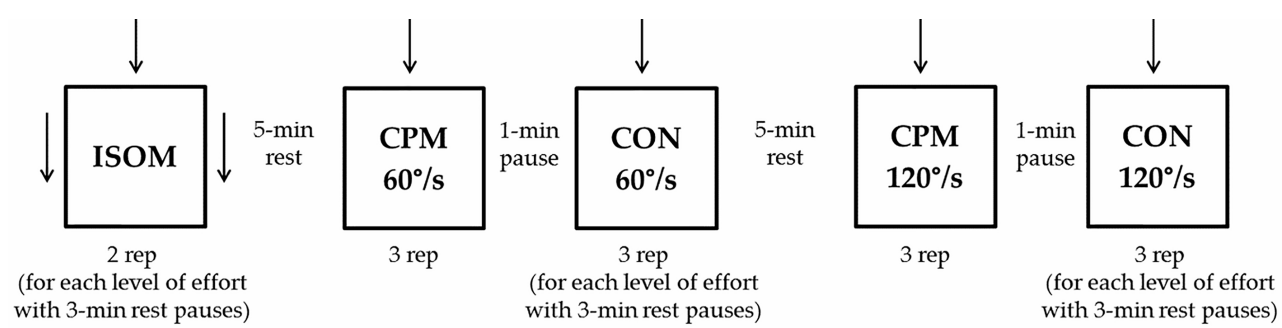

Fig. 1. Schematic representation of the experimental protocol. Arrows represent doublet stimulation. ISOM: isometric contraction; CPM: continuous passive isokinetic motion; CON: isokinetic concentric contraction; rep: repetitions.

therapy was selected (in order to avoid the risk for muscle hemorrhage). Their leg dominance was tested by means of the Waterloo footedness questionnairerevised [23]. All subjects gave written, informed consent before participating. The study was approved by the local Ethic committee of the Institution. The study conformed with the Code of Ethics of the World Medical Association (Declaration of Helsinki, British Medical Journal, 1964) for medical research involving human participants.

\subsection{Procedures}

\subsubsection{General}

The relationship between VA estimated through ITT and moments produced in ISOM and CON contractions at various levels of effort during knee extension were investigated. All tests were performed on the dominant lower limb. Subjects were instructed to exert five different levels of effort between $100 \%$ and $20 \%$ maximal voluntary contraction (MVC), differing by $20 \%$, in ISOM contractions at $50^{\circ}$ knee flexion, and in CON contractions at 60 and $120^{\circ}$ /s angular velocity.

\subsubsection{Familiarization}

In order to ease the test, subjects were accustomed to the unfamiliar motor task by performing 3 to 5 submaximal voluntary contractions for each condition and level of effort.

\subsubsection{Test sequencing}

For each level of effort, subjects performed two ISOM contraction tests, three CON60 contraction tests and three CON120 contraction tests. Timed 3-min rest periods separated each level of effort and 5-min rest periods separated each test. A subjectively maximal $(100 \%)$ effort was requested first. Submaximal efforts ( $80 \%$ to $20 \%$ ) were then required in decreasing order. This sequence was found to facilitate the elicitation of progressively decreasing efforts, closed to the re- quested ratio of the maximal effort, and their stability along the whole range of motion. For each subject, all tests were conducted in the same day. The rest pauses, in agreement with the literature, were deemed sufficient to prevent the onset of relevant fatigue [24], also considering the fact that most of the requested efforts were submaximal (Fig. 1).

\subsubsection{Moment recording}

During ISOM contractions, the extension moment was measured at $50^{\circ}$ knee flexion $\left(0^{\circ}=\right.$ full extension). During CON contractions, moment was measured instantaneously along the whole range of knee extension (about $100^{\circ}$ to $0^{\circ}$ ), at two constant angular velocities $\left(60^{\circ} / \mathrm{s}, 120^{\circ} / \mathrm{s}\right)$. All tests were performed using a Cybex Humac Norm ${ }^{\circledR} 2014$ isokinetic dynamometer (CSMi-Computer Sports Medicine, Inc; Stoughton, MA, USA). The subjects sat in an upright position with the hip flexed at about $90^{\circ}$ and grasped ad-hoc seat handles. The lateral epicondyle of the femur was aligned with the main horizontal axis of rotation of the dynamometer and the leg was secured to a Johnson anti-shear device [25]. Velcro straps were applied to the pelvis, the torso and the thigh, to secure the subject's body to the machine. For ISOM tests, resting twitches were recorded at $50^{\circ}$ knee extension, $3-5 \mathrm{~s}$ before contraction and 3-5 s after contraction. For CON tests, resting twitches were recorded at $50^{\circ}$ knee extension during continuous passive mobilization (CPM), before the active contraction tests: this implied a 3-4 min reset of the isokinetic ergometer software. The peak force of the pre-contraction twitch was adopted in the computation of VA (see Eq. (1) below). Indeed, the post-contraction RT may exhibit some potentiation because of the preceding contraction [26,27], so that preferring the pre-contraction twitch may lead in theory to underestimation of VA. However, various factors could counteract the effect of potentiation: a) post-contraction fatigue; b) fading of potentiation in ISOM tests that could be only assessed after a time- 
consuming reset of the ergometer; c) minor shortening/weakening of the Rectus femoris due to barely controllable anteversion (nutation) of the pelvis. In any case, tests were deemed as acceptable if the peak amplitude between pre- and post-contraction rest twitches differed by less than $\pm 10 \%$, which was invariably the case (not shown).

\subsubsection{Surface electromyography (EMG) recording}

In this study, surface EMG was recorded only to ascertain the relaxed state of the Vastus lateralis and of the homolateral Biceps femoris. Two pairs of silver chloride surface electrodes were applied on each muscle as per the SENIAM guidelines [28]. Low impedance $(<10 \mathrm{k} \Omega)$ of the skin-electrode interface was obtained by soaking and gently abrading the skin. The center-to-center interelectrode distance was $2 \mathrm{~cm}$. The reference electrode was applied to the contralateral patella. The EMG signals were recorded simultaneously with the moment signal and were used to evaluate the subject's resting state.

\subsubsection{Electrical stimulation}

VA level was determined by percutaneous electrical stimulation through two reusable wetted rubber electrodes. The anode was positioned medially on the anterior aspect of the upper thigh and the cathode was positioned medially on the anterior aspect of the lower thigh. The two electrodes $(16 \times 17 \mathrm{~cm}$ or $22 \times 17 \mathrm{~cm}$, depending on the subject's size) were secured to the thigh with a co-adhesive gauze. In this electrode montage, the stimulus reached the muscle fibers through peripheral nerve endings, and not through direct stimulation of the Femoral nerve trunk. The size of the electrodes warranted the stimulation of a representative portion of the Quadriceps. Through a constant current high-voltage stimulator (Digitimer ${ }^{\circledR}$ DS7A, Hertfordshire, UK), a doublet of single square-wave stimuli was delivered. This consisted in 2 square pulses, each with a $100-\mu$ s duration, $300 \mathrm{~V}$ maximal voltage, and intensity adjusted from 200 to $500 \mathrm{~mA}$, with a $10 \mathrm{~ms}$ interpulse interval. The intensity of the stimulation was determined as follows. The subject was asked to produce a MVC in one ISOM contraction at $50^{\circ}$ knee flexion for about $3 \mathrm{~s}$, and the peak moment was recorded. In successive bouts, the stimulus intensity was increased until at least $25 \%$ of MVC was recorded. This intensity was subsequently retained throughout the testing session.

\subsubsection{ISOM contractions testing}

Subjects were asked to perform ISOM contractions at various levels of subjective effort (see below). A first stimulus was delivered with the muscle fully relaxed (resting twitch, RT). Relaxation of Vastus lateralis and Biceps femoris was confirmed by the absence of surface EMG signals. Then, subjects were requested to produce maximal voluntary knee extensions until instructed to relax, about $5 \mathrm{~s}$ after a force plateau was reached. The stimulation was manually triggered within 2-3 s after the start of each contraction during a force plateau (interpolated twitch, IT). Within 3-6 s following each contraction, a third stimulus was delivered to the muscle at complete rest.

\subsubsection{CON contractions testing}

Subjects were asked to perform voluntary knee extensions at two constant angular velocities $\left(60^{\circ} / \mathrm{s}\right.$ and $120 \%$ s) and various levels of effort (see below). The movement started with the knee held at about $100^{\circ}$ and ended at about $0^{\circ}$, depending on subject's anatomical constraints. Subjects were asked to maintain the requested effort throughout the entire range of motion. No resistance to the following flexion was opposed by the isokinetic lever.

In order to measure the RT the leg was passively moved by the machine lever through the same range of motion at the same angular velocity (continuous passive motion, CPM).

Through a customized software routine, the instant of stimulation was computed in order to make the moment of the interpolated twitch to peak at exactly $50^{\circ}$ knee angle (IT). Preliminary data evidenced that the peak moment was reached about $100 \mathrm{~ms}$ and $50 \mathrm{~ms}$ after the doublet was released, during CPM and CON contractions, respectively, as expected by the delay imposed by muscle conduction velocity, excitationcontraction coupling, and tendon stiffness (data not shown). The accuracy of this empirical estimation was verified and adjusted on every single subject.

\subsubsection{Data processing and analysis}

A signal acquisition system (CED 1401, Cambridge Electronic Designed Limited-Ced, Cambridge, UK) was used to simultaneously record moment, knee angle, and angular velocity from the isokinetic dynamometer. Moment, knee angle, and angular velocity were sampled at $500 \mathrm{~Hz}$. A customized software (Spike 2, version 8, Cambridge Electronic Design Limited-CED, Cambridge, UK), connected to the acquisition system, controlled the electric stimulator in 

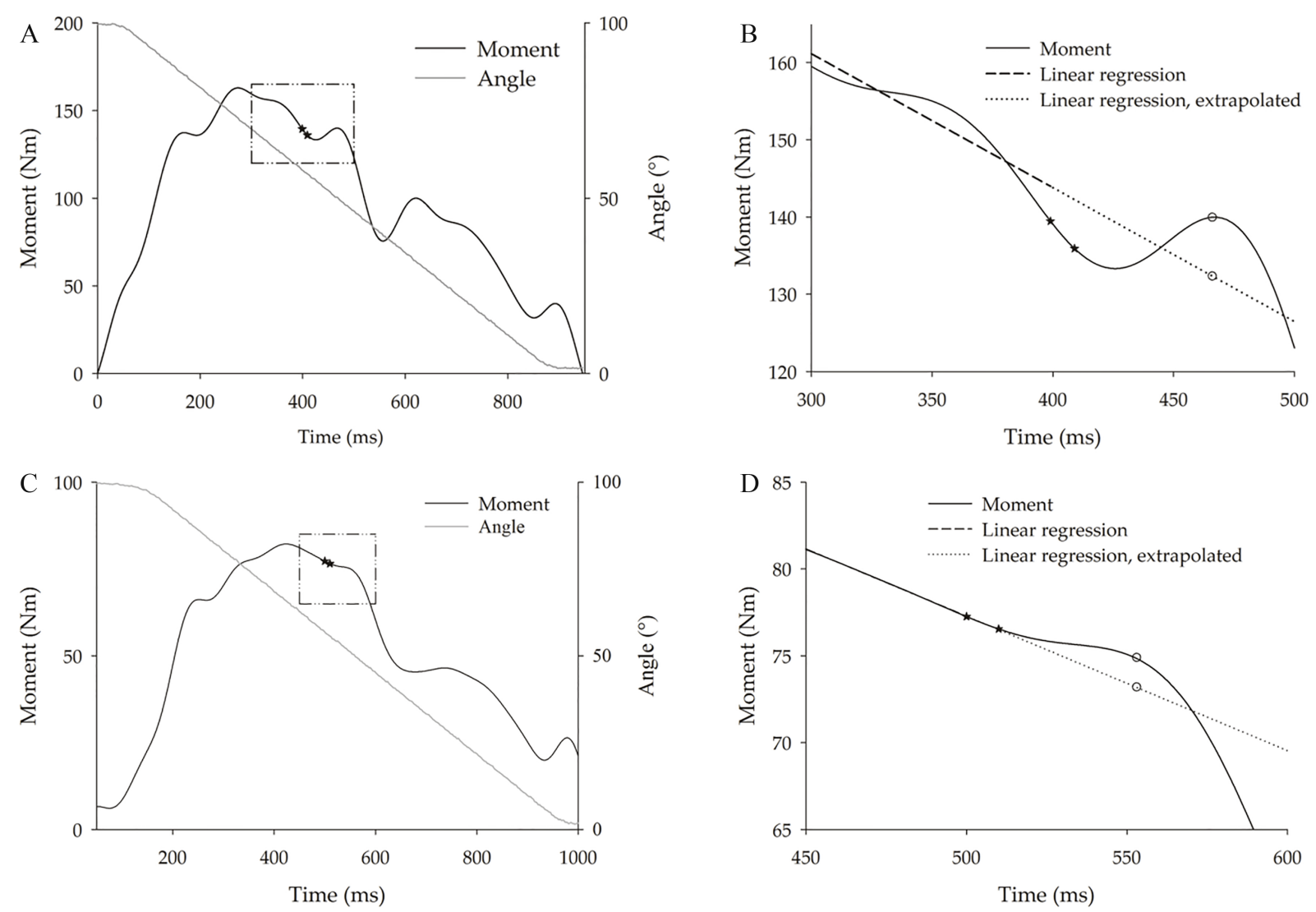

Fig. 2. Representative tracings of knee extension moment over time during (A and B) $60 \%$ and (C and D) $100 \%$ maximal voluntary CON120 contractions. Continuous black line: moment-time curve; stars: doublet of electrical stimuli. A) The continuous gray line represents the knee angle. B) The area encased by the square delimited by dash-dot line in panel A is expanded. The dashed black line gives the regression line of the voluntary moment computed from data recorded during the $100 \mathrm{~ms}$ preceding the stimulus; the dotted line represents its extrapolation, i.e. the estimated moment that would have occurred without stimulation. C) Example of a trial in which the stimulus did not determine a peak moment, but a transient flattening of the moment decay instead. D) The area encased by the square delimited by dash-dot segments in panel $\mathrm{C}$ is expanded. IT peak was computed as the highest distance between the observed (empty circle on the continuous black line) and the simultaneous extrapolated moment (empty circle on the dotted line).

order to elicit the stimulus at the desired knee angle. All data were off-line stored and processed. The average moment applied to the transducer with the lower limb fully extended was used to offset moment data at all angles, by proper computation. Moment signals were off-line filtered (low pass FIR filter, $1.3 \mathrm{~Hz}$ ).

VA was calculated using the following formula

$$
\mathrm{VA}=[1-(\mathrm{IT} / \mathrm{RT})] \%
$$

where IT (Interpolated Twitch) represents the increment in moment evoked during the contraction and $\mathrm{RT}$ represents the increment in moment evoked by the same stimulus during relaxation.

IT was computed as the difference between the visible moment increment following the delivery of the stimulus and the moment value at the instant of the delivery of the stimulus, during both rest and contraction.
During CON contractions, a bell-shaped momentangle relationship is observed, peaking around $75-80^{\circ}$ knee flexion $\left(0^{\circ}=\right.$ full extension $)$, reflecting changes in muscle length and moment arm, and perhaps unsteady levels of activation. In our sample, as it is known from the literature, the peak moment occurred closer to full extension, the higher the angular velocity (not shown, $[13,29,30])$. This is not relevant here, given that at the preset angle $\left(50^{\circ}\right)$ the VA, not the moment, was of interest. In absence of a constant moment, the background level against which the twitch may emerge was estimated by linear extrapolation of the slope of the moment signal during the $100 \mathrm{~ms}$ prior to the delivery of the stimulus, provided the moment was monotonically decreasing. The IT could be calculated as the difference between the peak moment subsequent to the delivery of the stimulus and the extrapolated moment, 
Table 1

Demographic characteristics of the study participants. Values are means (SD)

\begin{tabular}{lccccc}
\hline & Age (years) & Height $(\mathrm{cm})$ & Weight $(\mathrm{kg})$ & BMI $\left(\mathrm{kg} / \mathrm{m}^{2}\right)$ & Isometric peak moment/weight $(\mathrm{Nm} / \mathrm{kg})^{*}$ \\
\hline Total $(n=22)$ & $25.7(5.6)$ & $172.9(9.3)$ & $64.8(11.2)$ & $21.5(2.3)$ & $2.45(0.41)$ \\
Women $(n=11)$ & $26.4(6.9)$ & $165.8(9.3)$ & $56.7(8.0)$ & $20.6(2.1)$ & $2.20(0.20)$ \\
Men $(n=11)$ & $25.1(4.1)$ & $180.0(6.6)$ & $72.9(7.4)$ & $22.5(2.1)$ & $2.70(0.43)$ \\
\hline
\end{tabular}

BMI: body mass index. *Weight-normalized isometric peak moments refer to 10 subjects ( 5 women and 5 men) who performed the isometric test.

at the same instant of the twitch peak. In case no sharp twitch-related peaks could be detected, but a transient decrease of the moment negative slope was recorded, the difference between the highest post-twitch and the extrapolated moment level was measured. The same method was used for the computation of the RT. The procedures for estimation of the IT are represented in Fig. 2A-D. In some cases, the background moment (hence, the IT) could not be validly estimated through linear regression, because of high irregularities of the curve and, most frequently, because of a delayed peak, at or beyond the target angle of $50^{\circ}$ flexion. In these cases, tracings were discarded.

Computations, statistics, and graphic representations were performed using MATLAB ${ }^{\mathrm{TM}}$ (MathWorks Inc., version 8, Natick, MA, USA), STATA ${ }^{\text {TM }}$ (STATA Corp., version 14.0, College Station, TX, USA), and SigmaPlot ${ }^{\mathrm{TM}}$ (Systat software Inc., version 14.0, San Jose, CA, USA) software.

\subsubsection{Normalization}

Moment values for each subject and contraction condition were expressed as a percentage of the maximal moment level estimated by the linear regression of the relationship between VA and moment.

\subsection{Statistical analyses}

A standard linear regression was performed to model the relationship between VA and moment for the three conditions (ISOM, CON60 and CON120 contractions, dummy-coded). In order to test the statistical equivalence of the regression lines, a multiple linear regression was conducted among VA, moment, contraction conditions (dummy-coded), and their interactions. In case of significant models, a Tukey's post-hoc test was run on contrasts between pairs of conditions. The level of significance for all statistical tests was set at $p<$ 0.05 .

\section{Results}

Twenty-two healthy volunteers (11 women and 11 men), with a mean (standard deviation, SD) age of 25.7 (5.6) yr., a mean (SD) body mass of 64.8 (11.2) kg, and a mean (SD) height of $172.9(9.3) \mathrm{cm}$, (Table 1) were enrolled in the study (see Methods).

The relationship between VA and moment during ISOM and CON contractions at 60 and $120 \%$ s for different levels of effort, performed by 10 subjects for each condition, is shown in Figs 3-5. A linear fit was obtained for each contraction condition $(p=0.00)$. Across the subjects, the slopes of the VA-moment regression lines were (mean $(95 \%$ confidence intervals)) 1.04 (0.98-1.11), 0.96 (0.89-1.02), 0.84 (0.78$0.91)$, and the intercepts were $-5.22(-8.61--1.83)$, 4.16 (0.71-7.62), and 14.58 (10.76-18.39) for ISOM, CON60 and CON120 contractions, respectively. After multiple regression analysis, significant contrasts Tukey's (post-hoc) emerged across results obtained in CON120 contractions and the other two conditions ( $p=0.00$ and $p=0.02$, with respect to ISOM and CON60 contractions, respectively).

\section{Discussion}

The sample size of the present study is in agreement with previous studies on isometric [7,12,31], and isokinetic testing [17], based on 6-10 subjects. The validity of the ITT itself as an index of voluntary activation has been challenged on different grounds: criticisms arose from biomechanical considerations (e.g. the bias introduced by muscular fatigue, the risk for coactivation of antagonists, the risk for antidromic collision of the superimposed shock with the descending volitional drive) and from strictly methodological problems (the pros and cons of multiple shocks, and the inconsistencies across twitches evoked by electrical stimulation of the nerve trunk or by magnetic stimulation of the motor cortex). All considered, consensus exists that ITT still retains its overall validity as an index of VA, although the need for skill in execution and experience in interpretation cannot be overemphasized [8,32].

Most of the current physiological debate, however, turns on isometric contractions. The scarce previous studies on VA during CON contractions provided con- 


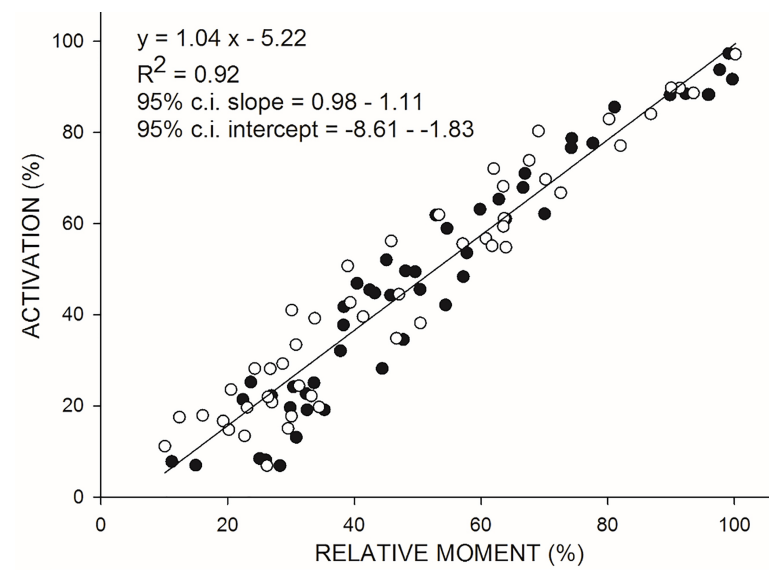

Fig. 3. Relationship between VA (on the ordinate) and moment (on the abscissa) during isometric (ISOM) contractions. Moment values were standardized with respect to the individual estimated maximal moment. White and black dots refer to each of the two trials performed by 5 women and 5 men, respectively. Data were best fitted by linear regression equation (see parameters given on the top right of the figure).

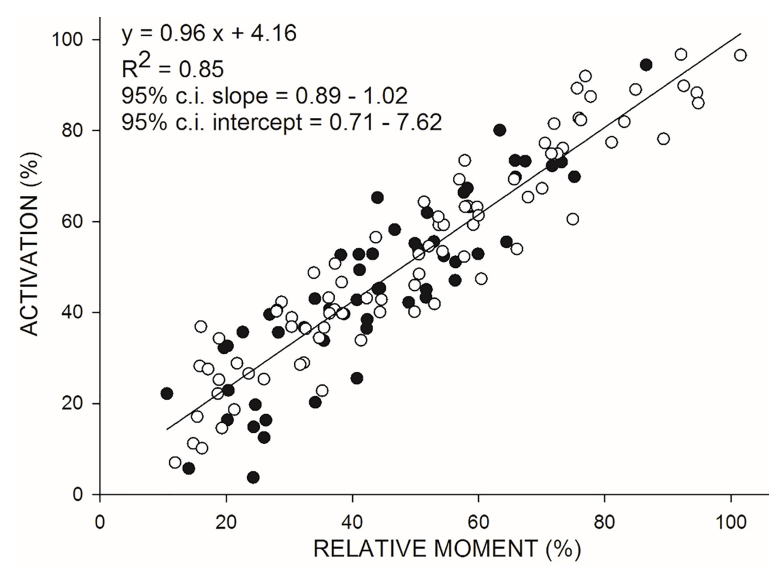

Fig. 4. Relationship between VA (on the ordinate) and relative moment (on the abscissa) during isokinetic concentric (CON60) contractions at $60^{\circ} \%$ s. Moment values were standardized with respect to the individual estimated maximal moment. White and black dots refer to each of the three trials performed by 5 women and 5 men, respectively. Data were best fitted by linear regression equation (see parameters given on the top right of the figure).

flicting results. Some authors observed that during maximal CON contractions at both slow $\left(20^{\circ} / \mathrm{s}\right)$ and fast angular velocities (150 and $300^{\circ} / \mathrm{s}$ ) VA was similar to that recorded during ISOM contractions $[9,16]$. On the contrary, others found that VA during $20 \%$ maximal CON contractions was lower compared to maximal ISOM contractions [17]. These discrepancies could arise from the different experimental methods. For example, different studies adopted single stimuli or

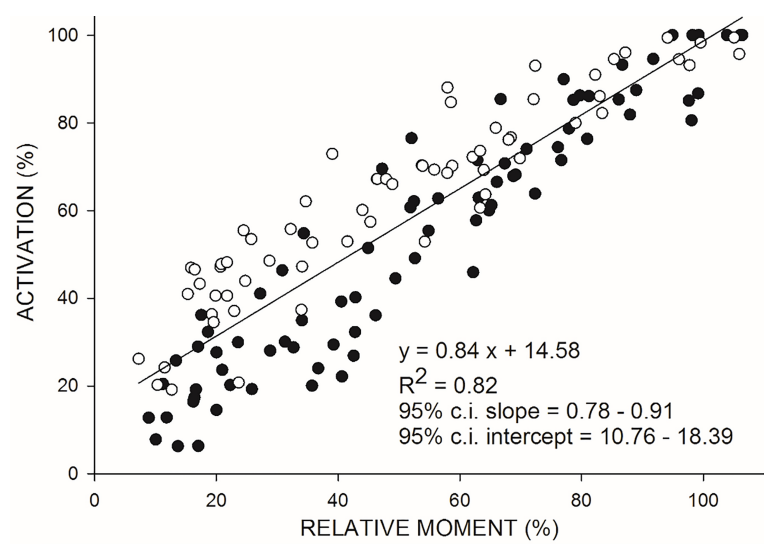

Fig. 5. Relationship between VA (on the ordinate) and relative moment (on the abscissa) during isokinetic concentric (CON120) contractions at $120 \%$ s. Moment values were standardized with respect to the individual estimated maximal moment. White and black dots refer to each of the three trials performed by 5 women and 5 men, respectively. Data were best fitted by linear regression equation (see parameters given on the top right of the figure).

short trains of 2 to 5 stimuli. Trains have been claimed to counteract the underestimation and the variability of twitch force [33]. Indeed, the first stimulus possibly goes partly "wasted" to increase the muscle's fiber pennation angle [29,31].

In the present study, the ITT generated by a doublet of electrical stimuli was adapted for application during CON contractions at 60 and $120 \%$ s. As expected, whatever the contraction condition, the IT peak decreased as moment increased. A linear relationship between VA and moment could be demonstrated for all contraction conditions (Figs 3-5), thus supporting the validity of the method. The VA-moment relationship for CON120 contractions was found to be significantly different with respect to the other two contraction conditions. The slope and the intercepts of the regression lines suggest that at low moment values VA was mildly underestimated (twitch peak higher than expected) during ISOM contractions and overestimated (twitch lower than expected, the more the higher the angular velocity) during CON contractions. It might be speculated that during ISOM contractions at low levels of effort the stimulus activates a sample of fibers which are not simultaneously activated voluntarily (and thus remain "free" to respond to the shock). During CON contractions at low levels of effort other factors may prevail. In general, the double stimulus may not fully counteract the architectural "slack" caused by the low pennation angle [34,35]. Also, the complex interplay between contractile and series elastic components may play a role $[3,13,35-38]$. In short, during CON con- 
tractions when the stimulus occurs the series elastic elements are still elongating, at a lower rate the lower the effort and the higher the speed (and thus, again, the lower the force). Correspondingly, the stimulus may make the contractile elements to "waste" their force against more compliant elastic elements: the lower the resulting twitch, the higher the estimate of VA.

Research on VA during CON contractions is scarce. One study applied the ITT technique to the investigation of the relationship between VA and peak moment during CON contractions at $20^{\circ} / \mathrm{s}$ and $150^{\circ} \%$, at $75^{\circ}$ knee flexion. This relationship emerged not to be statistically different between ISOM and CON contractions [16]. Other researchers measured the IT peak generated by a single electrical stimulus applied to the femoral nerve at a constant knee angle of $55^{\circ}$ during CON and eccentric contractions at $20 \%$ s. The slope of the VA-moment relationship for the eccentric condition was significantly lower than those obtained for ISOM and CON contractions, reflecting a lower neural drive and the preferential activation of weaker, highthreshold motor units [17]. The same Authors also studied the relationship between surface EMG activity (a proxy of VA) and VA, at both CON60 and CON120 contractions, during maximal and sub-maximal efforts. An exponential fit was demonstrated, with no velocitydependent differences [39].

The divergence across these few studies may be ascribed to methodological issues. At least two major technical difficulties may undermine the measurement of VA during CON contractions. First, the exact amplitude of twitches can be difficult to appreciate against a background force that, unlike in ISOM efforts, undergoes complex systematic changes. For instance, the estimation procedure (see Fig. 2) of the "basal" expected moment against which the twitch peak increment was computed in the present study was similar, but not equivalent, to that proposed in a previous article [17]. Secondly, the joint angle at which the twitch is peaking, and hence the instant of the stimulus delivery, must be carefully predetermined. Only few previous studies reported the use of a programmed delay in order to make the peak force to occur as synchronously as possible with respect to the desired knee angle $[16,40]$. On the contrary, the majority of researches reported the delivery of impulses to occur at a constant knee flexion angle whatever the angular velocity, which implies that the peak of the twitch force does not occur at the same angle [11,39].

The rate of force development (RFD) could be also an interesting index of validity of the method. The ca- pacity to modulate not only force but, also, its rate of development, is a fundamental property of neural control. This modulation is already possible at the level of a single motor unit [41]. Unfortunately, little is known on the RFD-activation relationship in our context. In the majority of past studies only maximal efforts were requested [42]. In addition, these were focused on RFD during isometric contractions with a few exceptions focusing on RFD during dynamic movements, in any case with maximal effort, only $[43,44]$. An isolated report suggested that RFD is rather constant once $30 \%$ or more of the maximal force is required [45].

The main limitation of the present study lies in the small range of velocities tested. These are presumably suitable for rehabilitation studies but lower than those achieved in some daily activities such as sit-up, and in many sports. Also, the assumption of data homoscedasticity in the linear regression model may not be fully met, given that the normalization of the moment values needed to achieve the subjects' comparability led to a ceiling effect. Moreover, the limited sample adopted, although hopefully sufficient to validate the method itself, is not sufficient to provide robust normative references for future clinical applications.

\section{Conclusions}

One of the main goals of the present study was to validate the method in view of future applications in sports and rehabilitation sciences. These are still very scarce, although some examples exist of measurement of VA during CON contractions in peripheral/muscular impairments [14], (i.e., evaluation of VA after anterior cruciate ligament reconstruction) and neuromuscular fatigue $[40,46]$. There is evidence that VA is suboptimal in many sport performances, and that it may decrease in various orthopedic and neurologic conditions, due to inhibition of peripheral origin and/or primitive limitations in the central neural drive. This deficit may be dependent on the velocity of muscle shortening [39]. Therefore future research might specifically aim at the development of training techniques targeting VA, both in sports and rehabilitation sciences. Proposals already exist. Some are of behavioral nature. These include plyometric exercises [47], motor imagery [48], and various forms of enhanced feedback capturing the EMG signal [49]. The most promising form of exercise is perhaps the EMG-triggered electrical stimulation of paretic muscles [50]. Other pioneering approaches are neurophysiological: this is the case for various forms 
of non-invasive electrical or magnetic brain stimulation, aimed at increasing the volitional drive after brain damage [51]. Such developments, in any case, require a reliable previous measurement of VA in both ISOM and CON contractions, and valid outcome measures. The method presented here may thus complement the functional assessment of force production and the validation of novel training and rehabilitation programs.

\section{Acknowledgments}

This research was funded by Istituto Auxologico Italiano, IRCCS, AMULET project, Ministry of Health, "Ricerca Corrente 2017".

\section{Conflict of interest}

There are no conflicts of interests.

\section{References}

[1] Gandevia SC. Spinal and supraspinal factors in human muscle fatigue. Physiol Rev 2001; 81: 1725-89.

[2] Molloy CB, Al-Omar AO, Edge KT, Cooper RG. Voluntary activation failure is detectable in some myositis patients with persisting quadriceps femoris weakness: An observational study. Arthritis Res Ther BioMed Central 2006; 8: R67.

[3] Osternig LR. Isokinetic dynamometry: Implications for muscle testing and rehabilitation. Exerc Sport Sci Rev 1986; 14 : 45-80.

[4] Massion J. Postural changes accompanying voluntary movements. Normal and pathological aspects. Hum Neurobiol 1984; 2: 261-7.

[5] Merton PA. Voluntary strength and fatigue. J Physiol 1954; 123: 553-64.

[6] Allen GM, Gandevia SC, McKenzie DK. Reliability of measurements of muscle strength and voluntary activation using twitch interpolation. Muscle Nerve 1995; 18: 593-600.

[7] Behm DG, St-Pierre DM, Perez D. Muscle inactivation: Assessment of interpolated twitch technique. J Appl Physiol 1996; 81: 2267-73.

[8] Taylor JL. Point: Counterpoint: The interpolated twitch does/does not provide a valid measure of the voluntary activation of muscle. J Appl Physiol 2009; 107: 354-5.

[9] Gandevia SC, Herbert RD, Leeper JB. Voluntary activation of human elbow flexor muscles during maximal concentric contractions. J Physiol 1998; 512: 595-602.

[10] Belanger AY, McComas AJ. Extent of motor unit activation during effort. J Appl Physiol 1981; 51: 1131-5.

[11] Babault N, Pousson M, Michaut A, Van Hoecke J. Effect of quadriceps femoris muscle length on neural activation during isometric and concentric contractions. J Appl Physiol 2003; 94: 983-90.

[12] Burke D, Hagbarth KE, Löfstedt L. Muscle spindle activity in man during shortening and lengthening contractions. J Physiol 1978; 277: 131-42.
[13] Kawakami Y, Kubo K, Kanehisa H, Fukunaga T. Effect of series elasticity on isokinetic moment-angle relationship in humans. Eur J Appl Physiol 2002; 87: 381-7.

[14] Otzel DM, Chow JW, Tillman MD. Long-term deficits in quadriceps strength and activation following anterior cruciate ligament reconstruction. Phys Ther Sport 2015; 16: 22-8.

[15] Shield A, Zhou S. Activation with the twitch interpolation technique. Sport Med 2004; 34: 253-67.

[16] Newham DJ, McCarthy T, Turner J. Voluntary activation of human quadriceps during and after isokinetic exercise. J Appl Physiol 1991; 71: 2122-6.

[17] Babault N, Pousson M, Ballay Y, Van Hoecke J. Activation of human quadriceps femoris during isometric, concentric, and eccentric contractions. J Appl Physiol 2001; 91: 2628-34.

[18] Bülow PM, Nørregaard J, Danneskiold-Samsøel B, Mehlsen $\mathrm{J}$. Twitch interpolation technique in testing of maximal muscle strength: Influence of potentiation, force level, stimulus intensity and preload. Eur J Appl Physiol Occup Physiol 1993; 67: 462-6.

[19] Nørregaard J, Lykkegaard JJ, Bülow PM, DanneskioldSamsøe B. The twitch interpolation technique for the estimation of true quadriceps muscle strength. Clin Physiol 1997; 17: 523-32.

[20] Roos MR, Rice CL, Connelly DM, Vandervoort AA. Quadriceps muscle strength, contractile properties, and motor unit firing rates in young and old men. Muscle Nerve 1999; 22: 1094-103.

[21] Robbins H. Some aspects of the sequential design of experiments. Bull Amer Math Soc 1952; 58: 527-35.

[22] Wald A. Sequential Analysis. New York: John Wiley Sons, Inc.; 1948.

[23] Elias LJ, Bryden MP, Bulman-Fleming MB. Footedness is a better predictor than is handedness of emotional lateralization. Neuropsychologia 1998; 36: 37-43.

[24] Willardson J. A brief review: Factors affecting the length of the rest interval between resistance exercise sets. J Strength Cond Res 2006; 20: 978-84.

[25] Li CK, Chan KM, Hsu SY, Chien P, Wong MW, Yuan Y. The Johnson antishear device and standard shin pad in the isokinetic assessment of the knee. Br J Sports Med 1993; 27 : 49-52.

[26] Kufel TJ, Pineda LA, Mador MJ. Comparison of potentiated and unpotentiated twitches as an index of muscle fatigue. Muscle Nerve 2002; 25: 438-44.

[27] Place N, Maffiuletti NA, Martin A, Lepers R. Assessment of the reliability of central and peripheral fatigue after sustained maximal voluntary contraction of the quadriceps muscle. Muscle Nerve 2007; 35: 486-95.

[28] Hermens HJ, Freriks B, Merletti R, Stegeman D, Blok J, Rau G, et al. European Recommendations for Surface ElectroMyoGraphy. Roessing Res Dev 1999.

[29] Folland JP, Williams AG. Methodological issues with the interpolated twitch technique. J Electromyogr Kinesiol 2007; 17: 317-27.

[30] Taylor NA, Cotter JD, Stanley SN, Marshall RN. Functional moment-velocity and power-velocity characteristics of elite athletes. Eur J Appl Physiol Occup Physiol 1991; 62: 116-21.

[31] Bampouras TM, Reeves ND, Baltzopoulos V, Maganaris CN. Muscle activation assessment: Effects of method, stimulus number, and joint angle. Muscle Nerve 2006; 34: 740-6.

[32] Horstman AM, Enoka RM, Girard O, Babault N, Herzog W, Racinais S, et al. Comments on Point: Counterpoint: The interpolated twitch does/does not provide a valid measure of 
the voluntary activation of muscle. J Appl Physiol 2009; 107: $359-66$.

[33] Suter E, Herzog W. Effect of number of stimuli and timing of twitch application on variability in interpolated twitch moment. J Appl Physiol 2001; 90: 1036-40.

[34] Fukunaga T, Ichinose Y, Ito M, Kawakami Y, Fukashiro S. Determination of fascicle length and pennation in a contracting human muscle in vivo. J Appl Physiol 1997; 82: 354-8.

[35] Reeves ND, Narici MV. Behavior of human muscle fascicles during shortening and lengthening contractions in vivo. J Appl Physiol 2003; 95: 1090-6.

[36] Bobbert MF, van Ingen Schenau GJ. Isokinetic plantar flexion: Experimental results and model calculations. J Biomech 1990; 23: 105-19.

[37] Harris RT, Dudley GA. Factors limiting force during slow, shortening actions of the quadriceps femoris muscle group in vivo. Acta Physiol Scand 1994; 152: 63-71.

[38] Ichinose Y, Kawakami Y, Ito M, Kanehisa H, Fukunaga T. In vivo estimation of contraction velocity of human vastus lateralis muscle during "isokinetic" action. J Appl Physiol 2000; 88: 851-6.

[39] Babault N, Pousson M, Michaut A, Ballay Y, Hoecke V. EMG activity and voluntary activation during knee-extensor concentric moment generation. Eur J Appl Physiol 2002; 86: 541-7.

[40] Babault N, Desbrosses K, Fabre M-S, Michaut A, Pousson M. Neuromuscular fatigue development during maximal concentric and isometric knee extensions. J Appl Physiol 2006; 100: 780-5.

[41] Baldissera F, Campadelli P. How motoneurones control development of muscle tension. Nature 1977; 268: 146-7.

[42] Maffiuletti NA, Aagaard P, Blazevich AJ, Folland J, Tillin N, Duchateau J. Rate of force development: Physiological and methodological considerations. Eur J Appl Physiol 2016; 116 : 1091-116.
[43] Tillin NA, Pain MTG, Folland JP. Contraction type influences the human ability to use the available moment capacity of skeletal muscle during explosive efforts. Proc R Soc B Biol Sci 2012; 279: 2106-15

[44] Adamson M, MacQuaide N, Helgerud J, Hoff J, Kemi OJ. Unilateral arm strength training improves contralateral peak force and rate of force development. Eur J Appl Physiol 2008; 103: 553-9.

[45] Rodríguez-Rosell D, Pareja-Blanco F, Aagaard P, GonzálezBadillo JJ. Physiological and methodological aspects of rate of force development assessment in human skeletal muscle. Clin Physiol Funct Imaging 2018; 38: 743-62.

[46] Cometti C, Deley G, Babault N. Effects of between-set interventions on neuromuscular function during isokinetic maximal concentric contractions of the knee extensors. J Sport Sci Med 2011; 10: 624-9.

[47] Behrens M, Mau-Moeller A, Mueller K, Heise S, Gube M, Beuster N, et al. Plyometric training improves voluntary activation and strength during isometric, concentric and eccentric contractions. J Sci Med Sport 2016; 19: 170-6.

[48] Grosprêtre S, Jacquet T, Lebon F, Papaxanthis C, Martin A. Neural mechanisms of strength increase after one-week motor imagery training. Eur J Sport Sci 2018; 18: 209-18.

[49] Schmidt RA, Lee TD. Augmented feedback. Human Kine Mot. Control Learn. A Behav. Emphas. Champaign, IL; 2005.

[50] Meilink A, Hemmen B, Seelen HAM, Kwakkel G. Impact of EMG-triggered neuromuscular stimulation of the wrist and finger extensors of the paretic hand after stroke: A systematic review of the literature. Clin Rehabil 2008; 22: 291-305.

[51] Kang N, Summers JJ, Cauraugh JH. Non-Invasive Brain Stimulation improves paretic limb force production: A systematic review and meta-analysis. Brain Stimul 2016; 9: 662-70. 